\title{
A novel consecutive three-component Heck-isomerization-Wittig sequence by way of in situ generated aldehydes
}

\author{
Jesco Panther, Adalbert Röhrich, and Thomas J. J. Müller* \\ Institut für Organische Chemie und Makromolekulare Chemie, Heinrich-Heine-Universität \\ Düsseldorf, Universitätsstraße 1, D-40225 Düsseldorf, Germany \\ E-mail: Thomasjj.Mueller@uni-duesseldorf.de
}

\section{Dedicated to Prof. Dr. Rainer Beckert on the occasion of his $60^{\text {th }}$ birthday}

\begin{abstract}
A novel consecutive three-component four step synthesis of 5-(hetero)arylpent-2-enoates has been disclosed. Various (hetero)aryl iodides can be coupled with allyl alcohol under Heck conditions to give 3-(hetero)arylpropionaldehyde intermediates, which were transformed without isolation with in situ generated stabilized phosphorus ylides to furnish 5-(hetero)arylpent-2enoates in moderate to excellent yield. This one-pot procedure circumvents the isolation of sensitive aldehydes and phosphorus ylides as intermediates and finally gives the product isomers with good to excellent $E / Z$ and $\beta / \alpha$ selectivity.
\end{abstract}

Keywords: Allyl alcohol, Heck reaction, multi-component reaction, one-pot reaction, Wittig reaction

\section{Introduction}

Multicomponent reactions (MCRs) are increasingly receiving interest in academia and industry. ${ }^{1,2}$ Besides addressing the fundamental principles of chemo-, regio- and stereoselectivity, MCRs are inherently efficient to a high degree. With respect to economical and ecological considerations conceptually novel synthetic approaches ${ }^{3,4}$ are directed towards the improvement of processes, ${ }^{5,6}$ simultaneously reducing the input of work and the output of waste. Therefore, MCRs are not only elegant but also beneficial for the environment. ${ }^{4}$ In particular, transition metal-catalyzed carbon-carbon bond forming processes have considerably enhanced the versatility of MCRs in construction of complex structural scaffolds and diversity oriented syntheses. ${ }^{7}$

Besides the huge class of Pd-catalyzed cross-coupling reactions, the mechanistically related Heck reaction ${ }^{8,9}$ is well received for tolerating many functional groups, most interestingly also aldehydes. This feature is particularly interesting, since reactive functionalities can be created 
during the aryl vinylation setting the stage for a subsequent transformation. Carbonyl olefination, e.g. by Wittig reaction with phosphorus ylides, is a highly chemo- and often stereo-selective methodology for the formation of double bonds. ${ }^{10}$

Just recently, two Heck-Wittig MCRs have been reported taking advantage of the extraordinary tolerance of aldehyde substrates in the first Heck step. ${ }^{11}$ However, even more intriguing would be the in situ generation of an aldehyde functionality and its subsequent olefination in a one-pot fashion. Here we report the development of a novel Heck-isomerizationWittig sequence as a tool for the rapid introduction of remote $\alpha, \beta$-unsaturated ester side chains of (hetero)arenes.

\section{Results and Discussion}

In most instances, the Heck reaction, as an aryl vinylation, furnishes styrene derivatives, if the $\beta$ hydride elimination is sterically and stereoelectronically feasible. Otherwise the newly established double bond is predictably formed in the sense of an allylation. However, a peculiar behavior was observed for allyl alcohols as substrates. ${ }^{12}$ According to the mechanistic rationale (Scheme 1) after olefine insertion placing the aryl substituent at the terminal carbon atom of the allyl system the $\beta$-hydride elimination product in direction of the hydroxy group furnishes a vinyl alcohol, which of course immediately tautomerizes to give an aldehyde. ${ }^{12}$ Therefore, this sequence can be named a Heck-isomerization reaction.

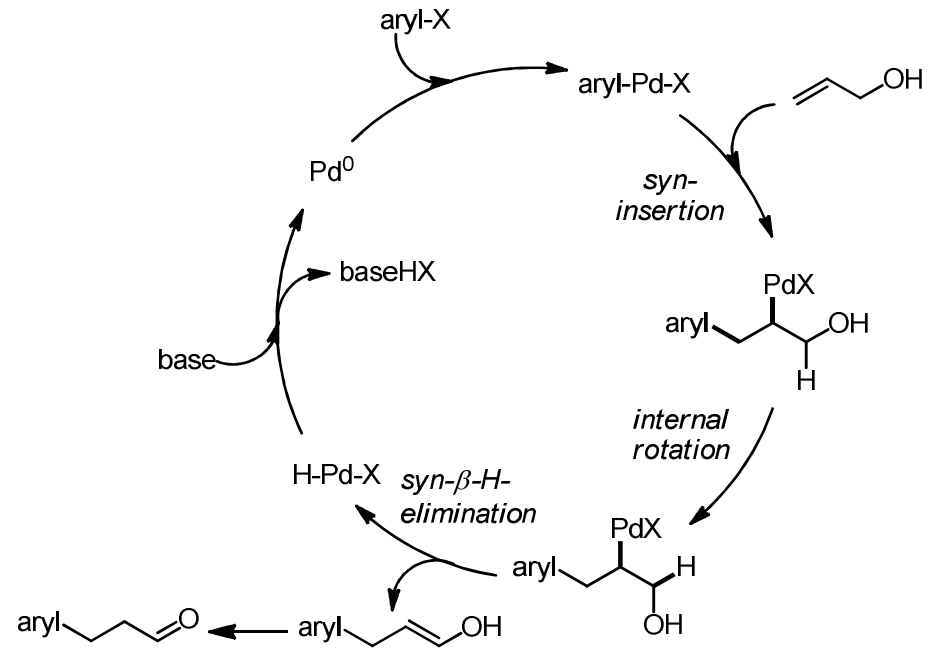

Scheme 1. Mechanistic rationale for the Heck-isomerization reaction with allyl alcohol as a substrate.

Surprisingly, the minor regioisomer of the insertion resulting in arylation at the central carbon atom of the allyl fragment also gives an aldehyde after $\beta$-hydride elimination. Thus, the 
two observed outcomes of the coupling under Heck condition result in the formation of an $\alpha$ and $\beta$-aryl propanal, the latter being the major product (Scheme 2).

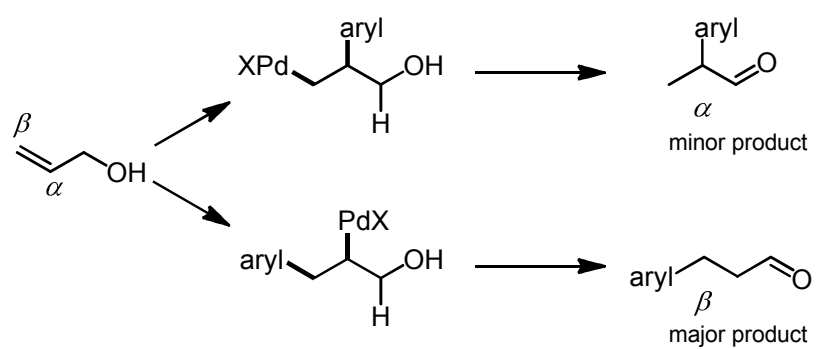

Scheme 2. Origin of $\alpha$ - and $\beta$-aryl propanal under Heck conditions for the reaction of aryl halides and allyl alcohol.

Since the Wittig reaction is one of the most powerful methodologies for the preparation of double bonds, ultimately from alkyl halides and carbonyls, ${ }^{10}$ we set out to concatenate the Heckisomerization reaction and the Wittig reaction to a novel one-pot process via the intermediacy of an aldehyde and an ylide (Scheme 3). With respect to previous experience from Pd-catalyzed insertion-cycloisomerization-Wittig ${ }^{13}$ and Rh-catalyzed Alder-ene cycloisomerization-Wittig ${ }^{14}$ sequences the planned combination appeared to be reasonable.

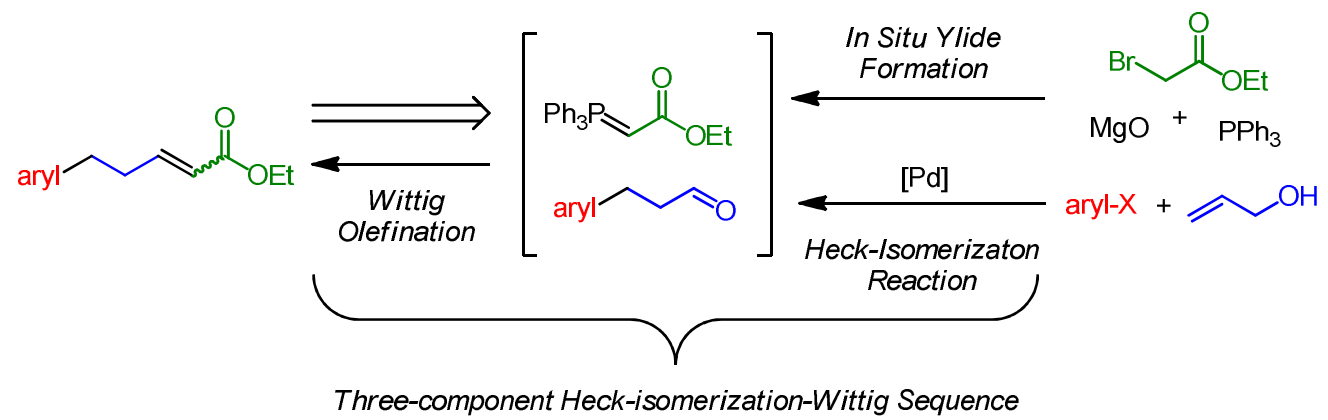

Scheme 3. Synthetic and retrosynthetic conception of a three-component Heck-isomerizationWittig sequence.

To provide higher convenience, where olefins can be directly generated from alkyl halides, carbonyls and phosphanes we decided to imply an in situ generation of the phosphonium salt and its conversion into an ylide in a one-pot fashion. ${ }^{15,16}$ Therefore, after reaction of iodobenzene (1a) and allyl alcohol (2) under Jeffery's conditions ${ }^{17}$ for $24 \mathrm{~h}$ at $30^{\circ} \mathrm{C}$ ethyl bromoacetate (3), triphenylphosphane, and magnesium oxide were successively added to smoothly promote the terminating carbonyl olefination at $30{ }^{\circ} \mathrm{C}$ within $12 \mathrm{~h}$. Ethyl 5-phenylpent-2-enoate (4a) was isolated in $91 \%$ yield as a mixture of E/Z-isomers (7:1) with a the regioselective formation of the $\beta$-isomer $(\beta / \alpha 13: 1)$ (Scheme 4; Table 1, Entry 1). 


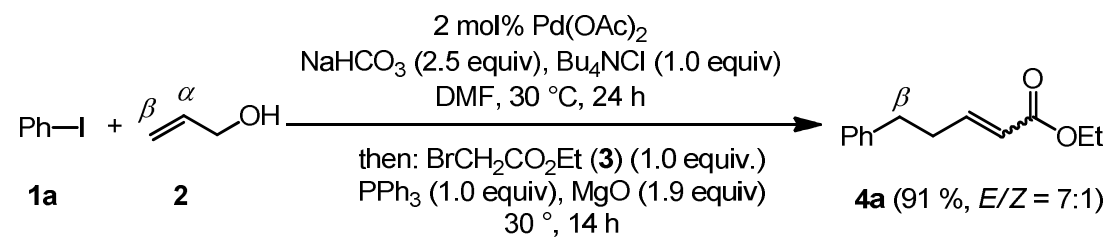

Scheme 4. Three-component Heck-isomerization-Wittig synthesis of ethyl 5-phenylpent-2enoate (4a) (only the $\beta$-regioisomer is depicted).

Although this initial attempt gave a remarkable efficiency with an average of $98 \%$ per step for the four-step one-pot sequence (Heck-isomerization, phosphonium salt generation, ylide formation, olefination) we sought to shorten the reaction times by an optimization study of the reaction temperatures of the Heck-isomerization and the Wittig steps (Scheme 5, Table 1).

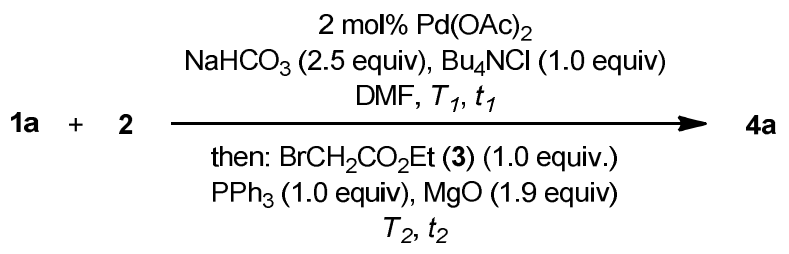

Scheme 5. Optimization of the reaction temperatures of Heck-isomerization-Wittig synthesis of ethyl 5-phenylpent-2-enoate (4a).

Starting from the initial conditions (Table 1, Entry 1) slightly elevated temperatures caused an acceleration of both steps and give rise to comparable isolated yields (Table 1, entries 2, 8 , and 9). However, increasing the reaction temperatures expectedly shortened the reaction times (as monitored by TLC), yet, the yields decreased substantially (Table 1, entries 3, 4, and 5). Either step might be carried out at higher temperature (Table 1, entries 6 and 7), however, not both steps (Table 1, entry 5). Conducting the reaction in a domino fashion, where all components were present from the very beginning, resulted in failure (Table 1, entry 10). This outcome was attributed to the inhibitory effect of phosphane on the catalytically active Pd species in the Heckisomerization step. As a compromise between short reaction times and high yields the conditions of entry 2 were finally chosen for testing the scope of the (hetero)aryl halides.

With these mild conditions for the sequence in hand, a novel three-component synthesis of 5arylpent-2-enoates 4 was established. After reaction of (hetero)aryl iodide $\mathbf{1}$ with allyl alcohol (2) under Jeffery's conditions for $6.5 \mathrm{~h}$ at $50{ }^{\circ} \mathrm{C}$ ethyl bromoacetate (3), triphenylphosphane, and magnesium oxide were successive added and after $1.5 \mathrm{~h}$ at $50{ }^{\circ} \mathrm{C}$ the ethyl 5-(hetero)arylpent-2enoates 4 were isolated in moderate to excellent yield (Scheme 6). ${ }^{18}$ The assigned structures of the compounds 4 were unambiguously supported by ${ }^{1} \mathrm{H}$ and ${ }^{13} \mathrm{C} N \mathrm{NR}$ spectroscopy, mass spectrometry and combustion analysis. 
Table 1. Optimization study of the reaction temperatures of the Heck-isomerization-Wittig sequence $^{\mathrm{a}}$

\begin{tabular}{cccccc}
\hline Entry & \multicolumn{2}{c}{ Heck-isomerization step } & \multicolumn{2}{c}{ Wittig step } & Ethyl 5-phenylpent-2-enoate $(\mathbf{4 a})^{\mathrm{b}}$ \\
\hline 1 & $T_{1}\left[{ }^{\circ} \mathrm{C}\right]$ & $t_{1}[\mathrm{~h}]$ & $T_{2}\left[{ }^{\circ} \mathrm{C}\right]$ & $t_{2}[\mathrm{~h}]$ & \\
\hline 2 & 30 & 24 & 30 & 14 & $91 \%$ \\
\hline 3 & 50 & 6.5 & 50 & 1.5 & $91 \%$ \\
4 & 60 & 5 & 60 & 1 & $85 \%$ \\
5 & 70 & 3 & 70 & 2 & $77 \%$ \\
6 & 100 & 0.5 & 100 & 0.3 & $59 \%$ \\
7 & 100 & 0.5 & 30 & 14 & $86 \%$ \\
8 & 30 & 24 & 100 & 0.3 & $87 \%$ \\
9 & 30 & 24 & 50 & 1.5 & $96 \%$ \\
$10^{\mathrm{c}}$ & 50 & 8 & 30 & 14 & $94 \%$ \\
\hline
\end{tabular}

${ }^{a}$ All reactions were carried out on a $1.00 \mathrm{mmol}$ scale with respect to iodobenzene (1a); Heck step: $\mathrm{Pd}(\mathrm{OAc})_{2}(0.02 \mathrm{mmol})$, allyl alcohol (2) (1.5 mmol), $\mathrm{NaHCO}_{3}(2.5 \mathrm{mmol}), \mathrm{Bu}_{4} \mathrm{NCl}$ $(1.0 \mathrm{mmol})$ in DMF $(3 \mathrm{~mL}, c(\mathbf{1 a})=0.3 \mathrm{M})$; Wittig step: ethyl bromoacetate $(1.0 \mathrm{mmol})$, triphenylphosphine $(1.0 \mathrm{mmol})$, and magnesium oxide $(1.9 \mathrm{mmol})$. All reaction times are the times needed for the complete consumption of the educt as indicated by TLC, exept otherwise stated. ${ }^{b}$ Yield after isolation and purification by column chromatography on silica gel as obtained as a mixture of $E / Z$-isomers (7:1) with a the regioselective formation of the $\beta$-isomer $(\beta / \alpha 13: 1)$.

${ }^{\mathrm{c}}$ All components were present from the very beginning, $T=30^{\circ} \mathrm{C}$ and $t=24 \mathrm{~h}$.

The final Wittig olefination step with the in situ generated stabilized phosphorus ylide furnishes $E / Z$ ratios ranging from 5:1 to 10:1 as supported by the appearance of the characteristic vicinal coupling constants of the double bond resonances in the ${ }^{1} \mathrm{H}$ NMR spectra. In case of the double olefination products $\mathbf{4 j}, \mathbf{4 o}$, and $\mathbf{4 p}$ the signal sets were significantly more complex, rendering the determination of the isomer ratios difficult.

As mentioned previously, the Heck reaction inherently leads to the formation of $\alpha$ - and $\beta$ substituted propenals, however, a clear selectivity in favor of the $\beta$-arylation product is obtained and the $\beta / \alpha$-product ratios range between 6:1 and 20:1. The characteristic doublet at $\delta 1.40$ has been used for the assignments of the $\alpha$-isomers. However, in some cases the ratio could not be determined due to overlapping signals. As exemplified for compound $\mathbf{4 f}$, the $E / Z$-isomers can be separated by simple column chromatography. However, the separation of $\beta / \alpha$-isomer mixtures are by far more challenging and has remained unsuccessful by flash chromatography so far. Therefore, future studies will be directed to increase the regioselectivity of the initial Heck step by careful ligand screening. 


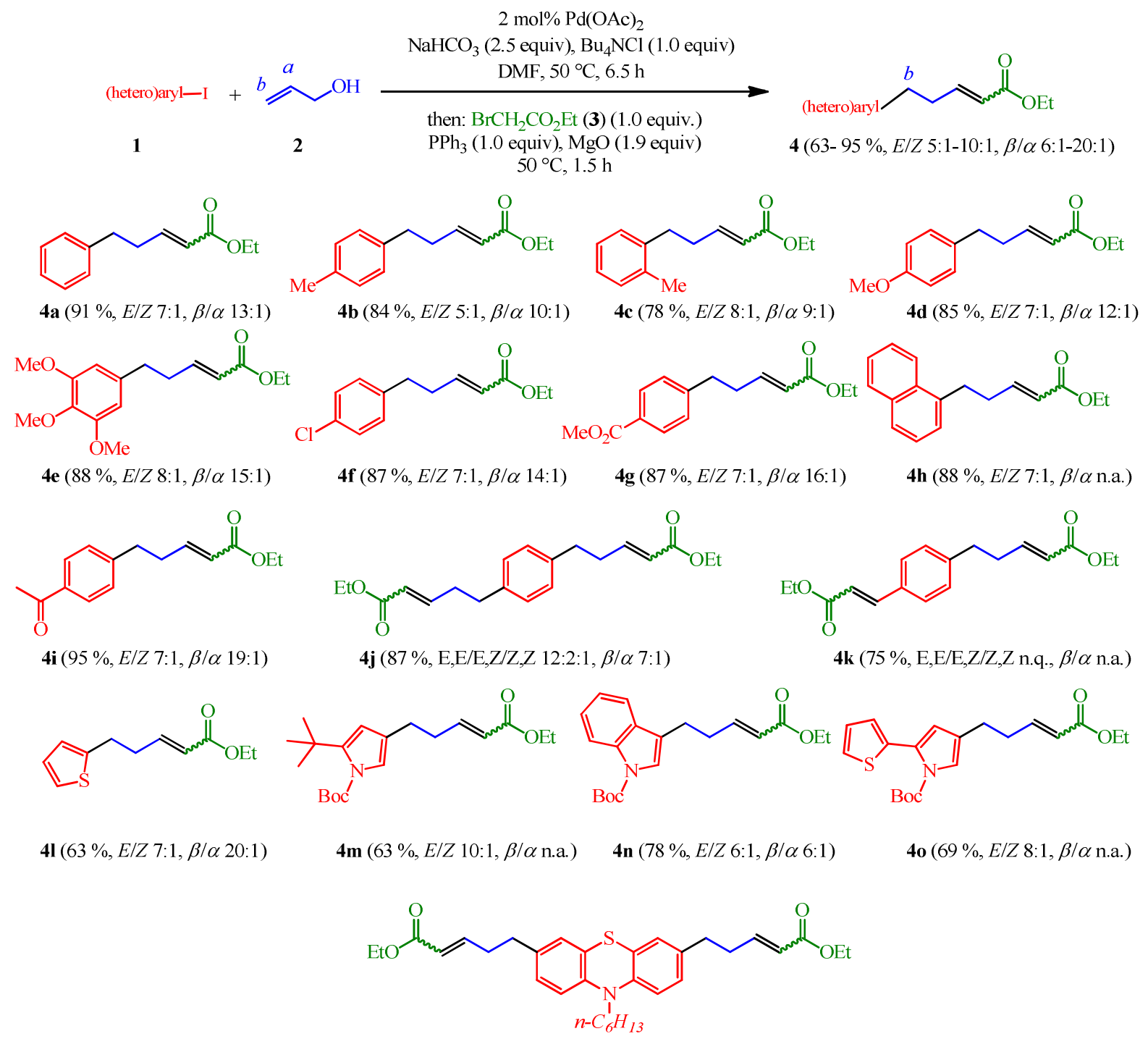

$4 \mathbf{p}(81 \%, E, E / E, Z / Z, Z 7: 1: 1, \beta / \alpha 14: 1)$

Scheme 6. Three-component Heck-isomerization-Wittig synthesis of 5-(hetero)arylpent-2enoates $4(\mathrm{Me}=$ methyl, Et $=$ ethyl, Boc $=$ tert-butoxycarbonyl, only the major $\beta$-regio isomers are depicted, n.a. $=$ not assignable by ${ }^{1} \mathrm{H}$ NMR). ${ }^{18}$

The product analysis of the substituent pattern of the 5-(hetero)arylpent-2-enoates 4 underlines that (hetero)aryl iodides 1 substituted by electron withdrawing (EWG), electron releasing (ERG) and electron neutral groups can be equally utilized in moderate to excellent yields. Substituents in the ortho (1c), meta, (1e) and para (1b) position of the benzene ring as well as multiple substituted phenyl derivatives, such as the trimethoxyphenyl moiety, are well tolerated. Polycyclic aromatic iodides (1h) are reacted without problems. A variety of functional groups, such as halide (1f), ketone (1i), ester (1j), carbamate (1n), and methoxy (1d) can be 
carried through the reaction sequence. A set of electron-rich heteroaryl substituents, which are occasionally problematic in transition metal catalysis, are transformed uneventfully (1l-p). Diiodoarenes (1j) and diiodoheteroarenes (1p) smoothly undergo double arylation and double olefination. Expectedly, the ketone substituted arene 2i displays chemoselectivity in favor of the aldehyde in the olefination step.

Some limitations are worth mentioning. Highly sterically hindered aryl iodides 1, such as 2,6-dimethoxy iodobenzene, failed to react in the sequence. All three regioisomeric (ortho, meta and para) iodo pyridines failed to react under the given conditions.

Since both intermediates, aliphatic aldehydes and phosphorus ylides are thermally sensitive to some extent, the major advantage of this sequence is their in situ generation. Furthermore, a methodological advantage of the sequence can be seen in the strictly stoichiometric use of only 1.0 equivalent of ethyl bromoacetate and triphenylphosphane, respectively, per aldehyde functionality. This not only renders the process economical, but also purification is significantly facilitated, dispensing of often tedious separation problems of phosphane oxide and byproducts resulting from excessive phosphorus ylids and triphenylphosphane. ${ }^{16}$

5-(Hetero)arylpent-2-enoates 4 are interesting intermediates in organic synthesis. They can be regarded as structural precursors for the preparation of numerous natural and pharmacological active compounds, such as kavalactones, ${ }^{19} \mathrm{GnRh}$ receptor antagonists, ${ }^{20}$ chromanes $^{21}$ dihydrochinoline $^{22}$, cryptophycins, ${ }^{23}$ eupolauramine, ${ }^{24}$ and HIV-1 protease inhibitors. ${ }^{25}$ As a consequence of the generation of an $\alpha, \beta$-unsaturated ester, 5-(hetero)arylpent-2-enoates 4 are versatile reaction partners for Michael reactions, ${ }^{26}$ dihydroxylations, ${ }^{27}$ cycloadditions, ${ }^{28}$ and for elaborations into allylic alcohols. ${ }^{29}$

\section{Conclusions}

In conclusion, we here disclosed a concise, efficient consecutive one-pot four-step sequence in the sense of a three-component Heck-isomerization-Wittig sequence for transforming (hetero)aryl iodides, allyl alcohol, and in situ generated stabilized phosphorus ylides into 5(hetero)arylpent-2-enoates. The mild reaction conditions are tolerated by many polar functional groups and the elaboration of this sequence into even more extended sequences apparently lies at hand. Further studies directed to improve the regioselectivity of the initial Heck step, to exploit and to expand the methodological scope of this sequence and the concatenation with other elementary processes are currently underway. 


\section{Experimental Section}

\section{General considerations}

All one-pot reactions were conducted in vacuum-dried Schlenk tubes under argon atmosphere. (Hetero)aryl iodides 1 were either purchased and used without further purification or they were prepared according to literature procedures (1m and 1o). ${ }^{30}$ Magnesium oxide light was purchased from Fluka. All reactions were monitored by TLC (silica gel 60, $\mathrm{F}_{254}$, Merck KGaA). The spots were detected with UV light at $\lambda_{\max , e x c}=254 \mathrm{~nm}$ and with aqueous potassium permanganate solution. Preparative flash column chromatography was conducted with silica gel $60(0.040$ $0.063 \mathrm{~mm}$, Merck KGaA). The crude mixtures were absorbed on Celite ${ }^{\circledR} 545$ (0.02-0.10 mm, Carl Roth $\mathrm{GmbH}$ Co.KG) prior to chromatographic purification. ${ }^{1} \mathrm{H},{ }^{13} \mathrm{C}$ and 135 -DEPT NMR spectra were recorded on a Bruker DRX 500 or Bruker AV III 300 in $\mathrm{CDCl}_{3}\left({ }^{1} \mathrm{H} \delta 7.26,{ }^{13} \mathrm{C} \delta\right.$ 77.0). The conventional abbreviations were used as follows: $s$ (singlet), $d$ (doublet), $t$ (triplet), $q$ (quartet), dt (doublet of triplets), m (multiplet). The EI spectra were recorded on a Finnigan ESQ 7000. Combustion analyses were carried out on a Perkin Elmer Series II Analyser 2400 in the Institut für Pharmazeutische und Medizinische Chemie at Heinrich-Heine-Universität Düsseldorf.

\section{General procedure of the three-component Heck-isomerization-Wittig sequence of 5- (hetero)arylpent-2-enoates (4)}

First, $\mathrm{Pd}(\mathrm{OAc})_{2}(4.50 \mathrm{mg}, 0.02 \mathrm{mmol}), \mathrm{NaHCO}_{3}(210 \mathrm{mg}, 2.50 \mathrm{mmol}), n \mathrm{Bu}_{4} \mathrm{NCl}(278 \mathrm{mg}$, $1.00 \mathrm{mmol})$ and dry, degassed DMF (3 mL) were placed in an argon flushed screw-cap Schlenk tube. Then, (hetero)aryl iodide $1(1.00 \mathrm{mmol})$ and allyl alcohol (2) $(1.5 \mathrm{mmol})$ were added (for experimental details, see Table 2). The mixture was placed in an oil bath at $50{ }^{\circ} \mathrm{C}$ and was stirred until the educt had disappeared as monitored by TLC. After cooling to room temperature, $\mathrm{MgO}$ (75.0 mg, $1.86 \mathrm{mmol}$ ), $\mathrm{PPh}_{3}$, (262 mg, $1.00 \mathrm{mmol}$ ) and ethyl bromoacetate $(167 \mathrm{mg}, 1.00 \mathrm{mmol})$ were subsequently added to the reaction mixture and was heated to $50{ }^{\circ} \mathrm{C}$ for the time $\mathrm{t}_{2}$. After completion of the sequence the crude reaction mixture was allowed to cool to room temperature and diluted with ethyl acetate and extracted three times with water. The organic phase was dried with anhydrous magnesium sulfate and adsorbed on Celite ${ }^{\circledR}$ during evaporation. Then, the product was purified by chromatography on silica gel ( $n$-hexane/ethyl acetate) to afford the analytically pure 5-(hetero)arylpent-2-enoates 4.

Ethyl 5-phenylpent-2-enoate (4a). Colorless oil. ${ }^{1} \mathrm{H}$ NMR (300 MHz, $\left.\mathrm{CDCl}_{3}\right)$ : $(E)$-isomer: $\delta$ $1.29\left(\mathrm{t},{ }^{3} J=7.1 \mathrm{~Hz}, 3 \mathrm{H}\right), 2.49-2.57(\mathrm{~m}, 2 \mathrm{H}), 2.76-2.81(\mathrm{~m}, 2 \mathrm{H}), 4.19$ (q, $\left.{ }^{3} J=7.1 \mathrm{~Hz}, 2 \mathrm{H}\right), 5.85$ $\left(\mathrm{dt},{ }^{3} J=1.6,15.7 \mathrm{~Hz}, 1 \mathrm{H}\right), 7.01\left(\mathrm{dt},{ }^{3} J=6.8,15.7 \mathrm{~Hz}, 1 \mathrm{H}\right), 7.17-7.23(\mathrm{~m}, 3 \mathrm{H}), 7.27-7.33$ (m, 2 $\mathrm{H})$; additional signals for the minor $(Z)$-isomer: $\delta 5.78\left(\mathrm{dt},{ }^{3} \mathrm{~J}=1.7,11.5 \mathrm{~Hz}, 1 \mathrm{H}\right), 6.24\left(\mathrm{dt},{ }^{3} \mathrm{~J}=\right.$ $7.5,11.5 \mathrm{~Hz}, 1 \mathrm{H})$; additional signal for the minor $\alpha$-arylated isomer: $\delta 1.43\left(\mathrm{~d},{ }^{3} J=7.0 \mathrm{~Hz}, 1\right.$ H). ${ }^{13} \mathrm{C}$ NMR $\left(75 \mathrm{MHz}, \mathrm{CDCl}_{3}\right): \delta 14.2\left(\mathrm{CH}_{3}\right), 33.9\left(\mathrm{CH}_{2}\right), 34.3\left(\mathrm{CH}_{2}\right), 60.2\left(\mathrm{CH}_{2}\right), 121.8(\mathrm{CH})$, $126.1(\mathrm{CH}), 128.3(\mathrm{CH}), 128.4(\mathrm{CH}), 140.9\left(\mathrm{C}_{\text {quat }}\right), 148.0(\mathrm{CH}), 166.6\left(\mathrm{C}_{\text {quat }}\right) . \mathrm{EI}-\mathrm{MS}(70 \mathrm{eV}$, 
m/z (\%)): $204\left(\mathrm{M}^{+}, 3\right), 159$ (12), 130 (25), 91 (100), 77 (2). Anal. calcd. for $\mathrm{C}_{13} \mathrm{H}_{16} \mathrm{O}_{2}$ (204.3): C 76.44, H 7.90; Found: C 76.26, H 7.92\%.

Table 2. Experimental details of the three-component Heck-isomerization-Wittig sequence for the synthesis of 5-(hetero)arylpent-2-enoates 4

\begin{tabular}{|c|c|c|c|c|c|}
\hline & & $\begin{array}{c}\text { Heck- } \\
\text { isomerization step }\end{array}$ & Wittig step & & \\
\hline Entry & $\begin{array}{l}\text { (hetero)aryl } 1 \\
{[\mathrm{mg}](\mathrm{mmol})}\end{array}$ & $t_{1}[\mathrm{~h}]$ & $t_{2}[\mathrm{~h}]$ & $\begin{array}{c}\text { eluent for } \\
\text { purification }^{\mathrm{a}}\end{array}$ & $\begin{array}{c}\text { 5-(hetero)arylpent- } \\
\text { 2-enoates } 4 \\
{[\mathrm{mg}](\text { yield })}\end{array}$ \\
\hline 1 & $204(1.00) \mathbf{1 a}$ & 6.5 & 1.5 & $50 / 1$ & $185(91 \%)$ of $\mathbf{4 a}$ \\
\hline 2 & $220(1.00) \mathbf{1 b}$ & 6.5 & 1.5 & $20 / 1$ & $183(84 \%)$ of $\mathbf{4 b}$ \\
\hline 3 & $218(1.00) \mathbf{1 c}$ & 6.5 & 1.5 & $50 / 1$ & $170(78 \%)$ of $\mathbf{4 c}$ \\
\hline 4 & $235(1.00) \mathbf{1 d}$ & 7 & 1.5 & $50 / 1$ & $199(85 \%)$ of $\mathbf{4 d}$ \\
\hline 5 & $294(1.00) \mathbf{1 e}$ & 6.5 & 1.5 & $50 / 1$ & $259(88 \%)$ of $\mathbf{4 e}$ \\
\hline 6 & 238 (1.00) $\mathbf{1 f}$ & 8 & 14 & $50 / 1$ & $208(87 \%)$ of $\mathbf{4 f}$ \\
\hline 7 & $262(1.00) \mathbf{1 g}$ & 8.5 & 14 & $5 / 1$ & $228(87 \%)$ of $\mathbf{4 g}$ \\
\hline 8 & $254(1.00) \mathbf{1 h}$ & 8 & 14 & $50 / 1$ & $225(88 \%)$ of $\mathbf{4 h}$ \\
\hline 9 & $246(1.00) \mathbf{1 i}$ & 8.5 & 1.5 & $5 / 1$ & $235(95 \%)$ of $\mathbf{4 i}$ \\
\hline 10 & $329(1.00) \mathbf{1} \mathbf{j}^{\mathrm{b}}$ & 7 & 1.5 & $50 / 1$ & $287(87 \%)$ of $\mathbf{4 j}$ \\
\hline 11 & $113(0.50) \mathbf{1 k}^{\mathrm{c}}$ & 6.5 & 1.5 & $10 / 1$ & $114(75 \%)$ of $4 \mathbf{k}$ \\
\hline 12 & $210(1.00) 11$ & 24 & 1.5 & $20 / 1$ & $133(63 \%)$ of $4 \mathbf{l}$ \\
\hline 13 & 349 (1.00) 1m & 24 & 1.5 & $40 / 1$ & $221(63 \%)$ of $\mathbf{4 m}$ \\
\hline 14 & $343(1.00) 1 n$ & 11.5 & 14 & $20 / 1$ & $268(78 \%)$ of $4 \mathbf{n}$ \\
\hline 15 & $375(1.00) 10$ & 8 & 14 & $30 / 1$ & $258(69 \%)$ of $\mathbf{4 o}$ \\
\hline 16 & $535(1.00) \mathbf{1} \mathbf{p}^{\mathrm{b}}$ & 9 & 1.5 & $10 / 1$ & $436(81 \%)$ of $\mathbf{4 p}$ \\
\hline
\end{tabular}

${ }^{\mathrm{a}}$ n-hexane/ethyl acetate. ${ }^{\mathrm{b}}$ Two equivalents of the reaction components exept for (hetero)aryl $\mathbf{1}$ for both steps. ${ }^{\mathrm{c}}$ Two equivalents of the reaction components for the Wittig step.

Ethyl 5-(p-tolyl)pent-2-enoate (4b). Colorless oil. ${ }^{1} \mathrm{H}$ NMR (300 $\left.\mathrm{MHz}, \mathrm{CDCl}_{3}\right)$ : $(E)$-isomer: $\delta$ $1.29\left(\mathrm{t},{ }^{3} J=7.1 \mathrm{~Hz}, 3 \mathrm{H}\right), 2.32(\mathrm{~s}, 3 \mathrm{H}), 2.46-2.54(\mathrm{~m}, 2 \mathrm{H}), 2.71-2.77$ (m, $\left.2 \mathrm{H}\right), 4.19$ (q, ${ }^{3} J=7.1$ $\mathrm{Hz}, 2 \mathrm{H}), 5.85$ (dt, $\left.{ }^{3} J=1.6,15.6 \mathrm{~Hz}, 1 \mathrm{H}\right), 7,00\left(\mathrm{dt},{ }^{3} J=6.8,15.7 \mathrm{~Hz}, 1 \mathrm{H}\right), 7.06-7.15(\mathrm{~m}, 4 \mathrm{H})$; additional signals for the minor (Z)-isomer: $\delta 5.77\left(\mathrm{dt},{ }^{3} \mathrm{~J}=1.7,11.5 \mathrm{~Hz}, 1 \mathrm{H}\right), 6.23\left(\mathrm{dt},{ }^{3} \mathrm{~J}=7.4\right.$, $11.5 \mathrm{~Hz}, 1 \mathrm{H})$; additional signal for the minor $\alpha$-arylated isomer: $\delta 1.41\left(\mathrm{~d},{ }^{3} J=7.0 \mathrm{~Hz}, 1 \mathrm{H}\right)$. ${ }^{13} \mathrm{C}$ NMR $\left(75 \mathrm{MHz}, \mathrm{CDCl}_{3}\right): \delta 14.2\left(\mathrm{CH}_{3}\right), 21.0\left(\mathrm{CH}_{3}\right), 33.9\left(\mathrm{CH}_{2}\right), 34.0\left(\mathrm{CH}_{2}\right), 60.2\left(\mathrm{CH}_{2}\right)$, $\left.121.7(\mathrm{CH}), 128.2(\mathrm{CH}), 129.1(\mathrm{CH}), 135.6\left(\mathrm{C}_{\text {quat }}\right), 137.7\left(\mathrm{C}_{\text {quat }}\right), 148.2 \mathrm{CH}\right), 166.6\left(\mathrm{C}_{\text {quat }}\right)$. EIMS (70 eV, m/z (\%)): $218\left(\mathrm{M}^{+}, 4\right), 173$ (8), 144 (12), 105 (100), 77 (5). Anal. calcd. for $\mathrm{C}_{14} \mathrm{H}_{18} \mathrm{O}_{2}$ (218.3): C 77.03, H 8.31; Found: C 77.03, H 8.19\%. 
Ethyl 5-(o-tolyl)pent-2-enoate (4c). Colorless oil. ${ }^{1} \mathrm{H}$ NMR (300 $\left.\mathrm{MHz}, \mathrm{CDCl}_{3}\right)$ : $(E)$-isomer: $\delta$ $1.30\left(\mathrm{t},{ }^{3} J=7.1 \mathrm{~Hz}, 3 \mathrm{H}\right), 2.31(\mathrm{~s}, 3 \mathrm{H}), 2.44-2.52(\mathrm{~m}, 2 \mathrm{H}), 2.74-2.79$ (m, $\left.2 \mathrm{H}\right), 4.18$ (q, ${ }^{3} J=7.1$ $\mathrm{Hz}, 2 \mathrm{H}), 5.88\left(\mathrm{dt},{ }^{3} J=1.6,15.6 \mathrm{~Hz}, 1 \mathrm{H}\right), 7.04\left(\mathrm{dt},{ }^{3} J=6.8,15.6 \mathrm{~Hz}, 1 \mathrm{H}\right), 7.11-7.18(\mathrm{~m}, 4 \mathrm{H})$; additional signals for the minor (Z)-isomer: $\delta 6.25\left(\mathrm{dt},{ }^{3} J=7.4,11.5 \mathrm{~Hz}, 1 \mathrm{H}\right)$; additional signal for the minor $\alpha$-arylated isomer: $\delta 1.41\left(\mathrm{~d},{ }^{3} J=7.0 \mathrm{~Hz}, 1 \mathrm{H}\right),{ }^{13} \mathrm{C} \mathrm{NMR}\left(75 \mathrm{MHz}, \mathrm{CDCl}_{3}\right): \delta 14.3$ $\left(\mathrm{CH}_{3}\right), 19.2\left(\mathrm{CH}_{3}\right), 31.7\left(\mathrm{CH}_{2}\right), 32.7\left(\mathrm{CH}_{2}\right), 60.2\left(\mathrm{CH}_{2}\right), 121.7(\mathrm{CH}), 126.1(\mathrm{CH}), 126.3(\mathrm{CH})$, $128.6(\mathrm{CH}), 130.3(\mathrm{CH}), 135.8\left(\mathrm{C}_{\text {quat }}\right), 139.0\left(\mathrm{C}_{\text {quat }}\right), 148.2(\mathrm{CH}), 166.6\left(\mathrm{C}_{\text {quat }}\right)$. EI-MS $(\mathrm{m} / z(\%))$ : $218\left(\mathrm{M}^{+}, 5\right), 173$ (10), 130 (20), 105 (100), 77 (6). Anal. calcd. for $\mathrm{C}_{14} \mathrm{H}_{18} \mathrm{O}_{2}$ (218.3): $\mathrm{C} 77.03, \mathrm{H}$ 8.31; Found: C 76.93, H 8.19\%.

Ethyl 5-(4-methoxyphenyl)pent-2-enoate (4d). Colorless oil. ${ }^{1} \mathrm{H}$ NMR (300 $\left.\mathrm{MHz}, \mathrm{CDCl}_{3}\right)$ : (E)Isomer: $\delta 1.28\left(\mathrm{t},{ }^{3} J=7.1 \mathrm{~Hz}, 3 \mathrm{H}\right), 2.45-2.53(\mathrm{~m}, 2 \mathrm{H}), 2.69-2.74(\mathrm{~m}, 2 \mathrm{H}), 3.79(\mathrm{~s}, 3 \mathrm{H}), 4.18$ $\left(\mathrm{q},{ }^{3} J=7.1 \mathrm{~Hz}, 2 \mathrm{H}\right), 5.83\left(\mathrm{dt},{ }^{3} J=1.6,15.6 \mathrm{~Hz}, 1 \mathrm{H}\right), 6.81-6.87(\mathrm{~m}, 2 \mathrm{H}), 6.99\left(\mathrm{dt},{ }^{3} J=6.8,15.7\right.$ $\mathrm{Hz}, 1 \mathrm{H}), 7.07-7.14(\mathrm{~m}, 2 \mathrm{H})$; additional signals for the minor $(Z)$-isomer: $\delta 6.22\left(\mathrm{dt},{ }^{3} J=7.4\right.$, $11.5 \mathrm{~Hz}, 1 \mathrm{H})$; additional signal for the minor $\alpha$-arylated isomer: $\delta 1.40\left(\mathrm{~d},{ }^{3} J=7.1 \mathrm{~Hz}, 1 \mathrm{H}\right) .{ }^{13} \mathrm{C}$ NMR (75 MHz, $\left.\mathrm{CDCl}_{3}\right): \delta 14.2\left(\mathrm{CH}_{3}\right), 33.4\left(\mathrm{CH}_{2}\right), 34.2\left(\mathrm{CH}_{2}\right), 55.2\left(\mathrm{CH}_{3}\right), 60.2\left(\mathrm{CH}_{2}\right), 113.9$ $(\mathrm{CH}), 121.8(\mathrm{CH}), 129.2(\mathrm{CH}), 132.9\left(\mathrm{C}_{\text {quat }}\right), 148.1(\mathrm{CH}), 158.0\left(\mathrm{C}_{\text {quat }}\right), 166.6\left(\mathrm{C}_{\text {quat }}\right)$. EI-MS (70 $\mathrm{eV}, \mathrm{m} / \mathrm{z}(\%)): 234\left(\mathrm{M}^{+}, 8\right), 189$ (4), 121 (100), 105 (10), 77 (6). Anal. calcd. for $\mathrm{C}_{14} \mathrm{H}_{18} \mathrm{O}_{3}$ (234.3): C 71.77, H 7.74; Found: C 71.53, H 7.66\%.

Ethyl 5-(3,4,5-trimethoxyphenyl)pent-2-enoate (4e). Yellow oil. ${ }^{1} \mathrm{H}$ NMR (300 $\left.\mathrm{MHz}, \mathrm{CDCl}_{3}\right)$ : (E)-isomer: $\delta 1.28\left(\mathrm{t},{ }^{3} \mathrm{~J}=7.1 \mathrm{~Hz}, 3 \mathrm{H}\right), 2.47-2.54(\mathrm{~m}, 2 \mathrm{H}), 2.69-2.74(\mathrm{~m}, 2 \mathrm{H}), 3.82(\mathrm{~s}, 3 \mathrm{H})$, $3.84(\mathrm{~s}, 6 \mathrm{H}), 4.18\left(\mathrm{q},{ }^{3} J=7.1 \mathrm{~Hz}, 2 \mathrm{H}\right), 5.85\left(\mathrm{dt},{ }^{3} J=1.6,15.7 \mathrm{~Hz}, 1 \mathrm{H}\right), 6.38(\mathrm{~s}, 2 \mathrm{H}), 6.99$ (dt, $\left.{ }^{3} J=15.6 \mathrm{~Hz}, 6.8 \mathrm{~Hz}, 1 \mathrm{H}\right)$; additional signals for the minor $(Z)$-isomer: $\delta 4.11$ (q, ${ }^{3} J=7.1 \mathrm{~Hz}, 2$ $\mathrm{H}), 5.78\left(\mathrm{dt},{ }^{3} \mathrm{~J}=1.6,11.5 \mathrm{~Hz}, 1 \mathrm{H}\right), 6.22\left(\mathrm{dt},{ }^{3} \mathrm{~J}=7.4,11.5 \mathrm{~Hz}, 1 \mathrm{H}\right)$; additional signal for the minor $\alpha$-arylated isomer: $\delta 1.41\left(\mathrm{~d},{ }^{3} J=7.0 \mathrm{~Hz}, 1 \mathrm{H}\right) \cdot{ }^{13} \mathrm{C} \mathrm{NMR}\left(75 \mathrm{MHz}, \mathrm{CDCl}_{3}\right): \delta 14.2$ $\left(\mathrm{CH}_{3}\right), 33.9\left(\mathrm{CH}_{2}\right), 34.8\left(\mathrm{CH}_{2}\right), 56.0\left(\mathrm{CH}_{3}\right), 60.2\left(\mathrm{CH}_{2}\right), 60.8\left(\mathrm{CH}_{3}\right), 105.2(\mathrm{CH}), 121.9(\mathrm{CH})$, $136.3\left(\mathrm{C}_{\text {quat }}\right), 136.5\left(\mathrm{C}_{\text {quat }}\right), 147.84(\mathrm{CH}), 153.14\left(\mathrm{C}_{\text {quat }}\right), 166.51\left(\mathrm{C}_{\text {quat }}\right)$. EI-MS (70 eV, $\left.\mathrm{m} / \mathrm{z}(\%)\right)$ : 294 ( $\left.\mathrm{M}^{+}, 16\right), 195$ (14), 181 (100), 91 (14). Anal. calcd. for $\mathrm{C}_{16} \mathrm{H}_{22} \mathrm{O}_{5}$ (394.3): C 65.29, H 7.53; Found: C 65.13, H 7.58\%.

Ethyl 5-(4-chlorophenyl)pent-2-enoate (4f). Colorless oil. ${ }^{1} \mathrm{H}$ NMR (300 $\left.\mathrm{MHz}, \mathrm{CDCl}_{3}\right)$ : $(E)$ isomer: $\delta 1.28\left(\mathrm{t},{ }^{3} J=7.1 \mathrm{~Hz}, 3 \mathrm{H}\right), 2.45-2.53(\mathrm{~m}, 2 \mathrm{H}), 2.72-2.77(\mathrm{~m}, 2 \mathrm{H}), 4.18\left(\mathrm{q},{ }^{3} J=7.1 \mathrm{~Hz}\right.$, $2 \mathrm{H}), 5.82\left(\mathrm{dt},{ }^{3} J=1.6,15.6 \mathrm{~Hz}, 1 \mathrm{H}\right), 6.96\left(\mathrm{dt}, J^{3}=6.8,15.7 \mathrm{~Hz}, 1 \mathrm{H}\right), 7.07-7.15(\mathrm{~m}, 2 \mathrm{H})$, 7.23-7.27 (m, $2 \mathrm{H}$ ); additional signals for the minor (Z)-isomer: $\delta 4.12\left(\mathrm{q},{ }^{3} J=7.1 \mathrm{~Hz}, 2 \mathrm{H}\right), 6.19$ $\left(\mathrm{dt},{ }^{3} J=7.5,11.5 \mathrm{~Hz}, 1 \mathrm{H}\right)$; additional signal for the minor $\alpha$-arylated isomer: $\delta 1.1\left(\mathrm{~d},{ }^{3} J=7.0\right.$ $\mathrm{Hz}, 1 \mathrm{H}) .{ }^{13} \mathrm{C}$ NMR $\left(75 \mathrm{MHz}, \mathrm{CDCl}_{3}\right): \delta 14.2\left(\mathrm{CH}_{3}\right), 30.2\left(\mathrm{CH}_{2}\right), 34.4\left(\mathrm{CH}_{2}\right), 69.9\left(\mathrm{CH}_{2}\right), 120.6$ $(\mathrm{CH}), 128.4(\mathrm{CH}), 129.8(\mathrm{CH}), 131.7\left(\mathrm{C}_{\text {quat }}\right), 139.6\left(\mathrm{C}_{\text {quat }}\right), 148.4(\mathrm{CH}), 166.2\left(\mathrm{C}_{\text {quat }}\right)$. EI-MS (70 $\mathrm{eV}, \mathrm{m} / \mathrm{z}(\%)): 240$ (1), $238\left(\mathrm{M}^{+}, 4\right), 193$ (8), 127 (34), 125 (100). Anal. calcd. for $\mathrm{C}_{13} \mathrm{H}_{15} \mathrm{ClO}_{2}$ (238.7): C 65.41, H 6.33; Found: C 65.28, H 6.49\%.

Methyl 4-(5-ethoxy-5-oxopent-3-en-1-yl)benzoate (4g). Yellow oil. ${ }^{1} \mathrm{H}$ NMR (300 MHz, $\mathrm{CDCl}_{3}$ ): (E)-isomer: $\delta 1.27\left(\mathrm{t},{ }^{3} \mathrm{~J}=7.1 \mathrm{~Hz}, 3 \mathrm{H}\right), 2.49-2.57(\mathrm{~m}, 2 \mathrm{H}), 2.80-2.85(\mathrm{~m}, 2 \mathrm{H}), 3.90$ (s, $3 \mathrm{H}), 4.17\left(\mathrm{q},{ }^{3} J=7.1 \mathrm{~Hz}, 2 \mathrm{H}\right), 5.82\left(\mathrm{dt},{ }^{3} J=1.6,15.6 \mathrm{~Hz}, 1 \mathrm{H}\right), 6.96\left(\mathrm{dt},{ }^{3} J=6.8,15.6 \mathrm{~Hz}, 1\right.$ 
$\mathrm{H}), 7.23-7.25(\mathrm{~m}, 2 \mathrm{H}), 7.95-7.98(\mathrm{~m}, 2 \mathrm{H})$; additional signal for the minor $(Z)$-isomer: $\delta 6.20$ (dt, $\left.{ }^{3} J=7.4,11.5 \mathrm{~Hz}, 1 \mathrm{H}\right)$; additional signal for the minor $\alpha$-arylated isomer: $\delta 1.44\left(\mathrm{~d},{ }^{3} J=7.0 \mathrm{~Hz}\right.$, $1 \mathrm{H}) .{ }^{13} \mathrm{C}$ NMR $\left(75 \mathrm{MHz}, \mathrm{CDCl}_{3}\right): \delta 14.2\left(\mathrm{CH}_{3}\right), 33.4\left(\mathrm{CH}_{2}\right), 34,3\left(\mathrm{CH}_{2}\right), 52.0\left(\mathrm{CH}_{3}\right), 60.2$ $\left(\mathrm{CH}_{2}\right), 122.2(\mathrm{CH}), 128.2\left(\mathrm{C}_{\text {quat }}\right), 128.4(\mathrm{CH}), 129.8(\mathrm{CH}), 146.1\left(\mathrm{C}_{\text {quat }}\right), 147.3(\mathrm{CH}), 166.4$ $\left(\mathrm{C}_{\text {quat }}\right), 167.0\left(\mathrm{C}_{\text {quat }}\right)$. EI-MS (70 eV, m/z $\left.(\%)\right): 262\left(\mathrm{M}^{+}, 4\right), 231$ (10), 216 (11), $188(16), 163$ (20), 149 (100), 121 (21). Anal. calcd. for $\mathrm{C}_{15} \mathrm{H}_{15} \mathrm{O}_{4}$ (262.3): $\mathrm{C} 68.68, \mathrm{H}$ 6.92; Found: $\mathrm{C}$ 68.48, $\mathrm{H}$ $6.65 \%$.

Ethyl 5-(naphthalen-2-yl)pent-2-enoate (4h). Colorless oil. ${ }^{1} \mathrm{H}$ NMR (300 MHz, $\left.\mathrm{CDCl}_{3}\right)$ : $(E)$ isomer: $\delta 1.30\left(\mathrm{t},{ }^{3} \mathrm{~J}=7.1 \mathrm{~Hz}, 3 \mathrm{H}\right), 2.62-2.71(\mathrm{~m}, 2 \mathrm{H}), 3.21-3.27(\mathrm{~m}, 2 \mathrm{H}), 4.19\left(\mathrm{q},{ }^{3} J=7.1 \mathrm{~Hz}\right.$, $2 \mathrm{H}), 5.91\left(\mathrm{dt},{ }^{3} \mathrm{~J}=1.6,15.7 \mathrm{~Hz}, 1 \mathrm{H}\right), 7.10\left(\mathrm{dt},{ }^{3} \mathrm{~J}=6.8,15.6 \mathrm{~Hz}, 1 \mathrm{H}\right), 7.32-7.56(\mathrm{~m}, 4 \mathrm{H}), 7.73-$ $7.75(\mathrm{~m}, 1 \mathrm{H}), 7.86-7.89(\mathrm{~m}, 1 \mathrm{H}), 7.99-8.02(\mathrm{~m}, 1 \mathrm{H})$; additional signals for the minor $(Z)-$ isomer: $\delta 5.81\left(\mathrm{dt},{ }^{3} J=1.6,11.5 \mathrm{~Hz}, 1 \mathrm{H}\right), 6.32\left(\mathrm{dt},{ }^{3} J=7.3,11.4 \mathrm{~Hz}, 1 \mathrm{H}\right) \cdot{ }^{13} \mathrm{C}$ NMR $(75 \mathrm{MHz}$, $\left.\mathrm{CDCl}_{3}\right): \delta 14.3\left(\mathrm{CH}_{3}\right), 31.4\left(\mathrm{CH}_{2}\right), 33.1\left(\mathrm{CH}_{2}\right), 60.2\left(\mathrm{CH}_{2}\right), 122.8(\mathrm{CH}), 123.4(\mathrm{CH}), 125.5(2$ $\mathrm{CH}), 125.9(\mathrm{CH}), 126.0(\mathrm{CH}), 127.0(\mathrm{CH}), 128.9(\mathrm{CH}), 131.6\left(\mathrm{C}_{\text {quat }}\right), 133.9\left(\mathrm{C}_{\text {quat }}\right), 136.8\left(\mathrm{C}_{\text {quat }}\right)$, $148.1(\mathrm{CH}), 166.6\left(\mathrm{C}_{\text {quat }}\right)$. EI-MS (70 eV, m/z (\%)): $254\left(\mathrm{M}^{+}, 10\right), 155$ (25), 141 (100), 130 (12), 105 (10), 115 (14), 91 (50). Anal. calcd. for $\mathrm{C}_{17} \mathrm{H}_{18} \mathrm{O}_{2}$ (254.3): $\mathrm{C} \mathrm{80.28,} \mathrm{H} \mathrm{7.13;} \mathrm{Found:} \mathrm{C}$ 80.08, H $7.21 \%$.

Ethyl 5-(4-acetylphenyl)pent-2-enoate (4i). Yellow oil. ${ }^{1} \mathrm{H} \mathrm{NMR}\left(300 \mathrm{MHz}, \mathrm{CDCl}_{3}\right)$ : $(E)$ isomer: $\delta 1.27\left(\mathrm{t},{ }^{3} J=7.1 \mathrm{~Hz}, 3 \mathrm{H}\right), 2.50-2.60(\mathrm{~m}, 2 \mathrm{H}), 2.58(\mathrm{~s}, 3 \mathrm{H}), 2.81-2.86(\mathrm{~m}, 2 \mathrm{H}), 4.18$ $\left(\mathrm{q},{ }^{3} J=7.1 \mathrm{~Hz}, 2 \mathrm{H}\right), 5.83\left(\mathrm{dt},{ }^{3} J=1.6,15.7 \mathrm{~Hz}, 1 \mathrm{H}\right), 6.96\left(\mathrm{dt},{ }^{3} J=6.8,15.6 \mathrm{~Hz}, 1 \mathrm{H}\right), 7.25-7.28$ $(\mathrm{m}, 2 \mathrm{H}), 7.87-7.91(\mathrm{~m}, 2 \mathrm{H})$; additional signal for the minor $(Z)$-isomer: $\delta 6.19\left(\mathrm{dt},{ }^{3} J=7.4,11.5\right.$ $\mathrm{Hz}, 1 \mathrm{H})$; additional signal for the minor $\alpha$-arylated isomer: $\delta 1.43\left(\mathrm{~d},{ }^{3} J=7.0 \mathrm{~Hz}, 1 \mathrm{H}\right) \cdot{ }^{13} \mathrm{C}$ NMR (125 MHz, $\left.\mathrm{CDCl}_{3}\right): \delta 14.2\left(\mathrm{CH}_{3}\right), 26.5\left(\mathrm{CH}_{3}\right), 33.3\left(\mathrm{CH}_{2}\right), 34.2\left(\mathrm{CH}_{2}\right), 60.2\left(\mathrm{CH}_{2}\right), 122.2$ $(\mathrm{CH}), 128.5(\mathrm{CH}), 128.6(\mathrm{CH}), 135.3\left(\mathrm{C}_{\text {quat }}\right), 146.4\left(\mathrm{C}_{\text {quat }}\right), 147.2(\mathrm{CH}), 166.4\left(\mathrm{C}_{\text {quat }}\right), 197.7$ $\left(\mathrm{C}_{\text {quat }}\right)$. EI-MS (70 eV, m/z (\%)): $246\left(\mathrm{M}^{+}, 10\right), 159$ (14), 133 (38), 130 (24), 105 (21), 91 (100). Anal. calcd. for $\mathrm{C}_{15} \mathrm{H}_{18} \mathrm{O}_{3}$ (246.3): C 73.15, H 7.37; Found: C 72.95, H 7.28\%.

Diethyl 5,5'-(1,4-phenylene)bis(pent-2-enoate) (4j). Colorless oil. ${ }^{1} \mathrm{H}$ NMR (300 MHz, $\left.\mathrm{CDCl}_{3}\right)$ : $(E, E)$-isomer: $\delta 1.28\left(\mathrm{t},{ }^{3} J=7.1 \mathrm{~Hz}, 6 \mathrm{H}\right), 2.46-2.54(\mathrm{~m}, 4 \mathrm{H}), 2.72-2.77(\mathrm{~m}, 4 \mathrm{H}), 4.18$ $\left(\mathrm{q},{ }^{3} J=7.1 \mathrm{~Hz}, 4 \mathrm{H}\right), 5.85\left(\mathrm{dt},{ }^{3} J=1.6,15.7 \mathrm{~Hz}, 2 \mathrm{H}\right), 6.99\left(\mathrm{dt},{ }^{3} J=6.8,15.7 \mathrm{~Hz}, 2 \mathrm{H}\right), 7.10(\mathrm{~s}, 4$ $\mathrm{H})$; additional signals for the minor $(E, Z)$-isomer: $\delta 1.28\left(\mathrm{dt},{ }^{3} \mathrm{~J}=7.1,6 \mathrm{H}\right), 2.46-2.54(\mathrm{~m}, 2 \mathrm{H})$, 2.71-2.76 (m, $4 \mathrm{H}), 2.93-3.01(\mathrm{~m}, 2 \mathrm{H}), 4.17(\mathrm{~m}, 4 \mathrm{H}), 5.77\left(\mathrm{dt},{ }^{3} J=1.6,11.5 \mathrm{~Hz}, 1 \mathrm{H}\right), 5.85(\mathrm{dt}$, $\left.{ }^{3} J=1.6,15.6 \mathrm{~Hz}, 1 \mathrm{H}\right), 6.23\left(\mathrm{dt},{ }^{3} J=7.4,11.5 \mathrm{~Hz}, 1 \mathrm{H}\right), 7.00\left(\mathrm{dt},{ }^{3} J=6.8,15.7 \mathrm{~Hz}, 1 \mathrm{H}\right), 7.08$ $7.16(\mathrm{~m}, 4 \mathrm{H})$; additional signals for the minor $(Z, Z)$-isomer: $\delta 1.28\left(\mathrm{t},{ }^{3} J=7.1 \mathrm{~Hz}, 6 \mathrm{H}\right), 2.45$ $2.53(\mathrm{~m}, 4 \mathrm{H}), 2.69-2.74(\mathrm{~m}, 4 \mathrm{H}), 4.18\left(\mathrm{q},{ }^{3} J=7.13 \mathrm{~Hz}, 4 \mathrm{H}\right), 5.82\left(\mathrm{dt},{ }^{3} J=1.6,15.7 \mathrm{~Hz}, 2 \mathrm{H}\right)$, 6.90-7.00 (m, $4 \mathrm{H}), 7.59-7.62(\mathrm{~m}, 2 \mathrm{H})$; additional signal for the minor $\alpha$-arylated isomer: $\delta 1.42$ $\left(\mathrm{d},{ }^{3} \mathrm{~J}=7.0 \mathrm{~Hz}, 1 \mathrm{H}\right) .{ }^{13} \mathrm{C} \mathrm{NMR}\left(75 \mathrm{MHz}, \mathrm{CDCl}_{3}\right): \delta 14.2\left(\mathrm{CH}_{3}\right), 33.9\left(2 \mathrm{CH}_{2}\right), 60.7\left(\mathrm{CH}_{2}\right), 121.8$ $(\mathrm{CH}), 128.4(\mathrm{CH}), 138.6\left(\mathrm{C}_{\text {quat }}\right), 148.1(\mathrm{CH}), 166.6\left(\mathrm{C}_{\text {quat }}\right)$. EI-MS (70 eV, $\left.\mathrm{m} / z(\%)\right): 330\left(\mathrm{M}^{+}, 4\right)$, 284 (14), 217 (34), 173 ( 54), 105 (100), 104 (32), 91 (14). Anal. calcd. for $\mathrm{C}_{20} \mathrm{H}_{26} \mathrm{O}_{4}$ (330.4): C 72.70, H 7.93; Found: C 72.79, H 8.71\%. 
Ethyl 5-(4-(3-ethoxy-3-oxoprop-1-en-1-yl)phenyl)pent-2-enoate (4k). Yellow oil. ${ }^{1} \mathrm{H}$ NMR (300 MHz, $\left.\mathrm{CDCl}_{3}\right)$ : $(E, E)$-isomer: $\delta 1.28\left(\mathrm{t},{ }^{3} J=7.1 \mathrm{~Hz}, 3 \mathrm{H}\right), 1.33\left(\mathrm{t},{ }^{3} J=7.1 \mathrm{~Hz}, 3 \mathrm{H}\right), 2.48$ $2.56(\mathrm{~m}, 2 \mathrm{H}), 2.77-2.82(\mathrm{~m}, 2 \mathrm{H}), 4.17$ (q, $\left.{ }^{3} J=7.1 \mathrm{~Hz}, 2 \mathrm{H}\right), 4.25\left(\mathrm{q},{ }^{3} J=7.1 \mathrm{~Hz}, 2 \mathrm{H}\right), 5.84$ (dt, $\left.{ }^{3} J=1.6,15.7 \mathrm{~Hz}, 1 \mathrm{H}\right), 6.40(\mathrm{~m}, 1 \mathrm{H}), 6.97\left(\mathrm{dt},{ }^{3} J=6.8,15.6 \mathrm{~Hz}, 1 \mathrm{H}\right), 7.18-7.21(\mathrm{~m}, 1 \mathrm{H}), 7.44-$ $7.47(\mathrm{~m}, 2 \mathrm{H}), 7.63-7.69(\mathrm{~m}, 2 \mathrm{H})$; additional signal for the minor $(E, Z) /(Z, E)$-isomer: $\delta 6.20(\mathrm{dt}$, $\left.{ }^{3} J=7.5,11.5 \mathrm{~Hz}, 1 \mathrm{H}\right)$; additional signals for the minor $(Z, Z)$-Isomer: $\delta 5.79\left(\mathrm{dt},{ }^{3} J=7.5,11.5\right.$ $\mathrm{Hz}, 1 \mathrm{H}), 6.21\left(\mathrm{dt},{ }^{3} J=1.5,11.5 \mathrm{~Hz}, 1 \mathrm{H}\right)$. additional signal for the minor $\alpha$-arylated isomer: $\delta$ $1.51\left(\mathrm{~d},{ }^{3} \mathrm{~J}=7.0 \mathrm{~Hz}, 1 \mathrm{H}\right) .{ }^{13} \mathrm{C} \mathrm{NMR}\left(75 \mathrm{MHz}, \mathrm{CDCl}_{3}\right): \delta 14.2\left(\mathrm{CH}_{3}\right), 14.3\left(\mathrm{CH}_{3}\right), 33.5\left(\mathrm{CH}_{2}\right)$, $34.2\left(\mathrm{CH}_{2}\right), 60.2\left(\mathrm{CH}_{2}\right), 60.4\left(\mathrm{CH}_{2}\right), 117.6(\mathrm{CH}), 122.1(\mathrm{CH}), 128.2(\mathrm{CH}), 128.9(\mathrm{CH}), 132.5$ $\left(\mathrm{C}_{\text {quat }}\right), 143.3\left(\mathrm{C}_{\text {quat }}\right), 144.3(\mathrm{CH}), 147.5(\mathrm{CH}), 166.5\left(\mathrm{C}_{\text {quat }}\right), 167.1\left(\mathrm{C}_{\text {quat }}\right) . \mathrm{EI}-\mathrm{MS}(70 \mathrm{eV}$, m/z (\%)): $302\left(\mathrm{M}^{+}, 5\right), 257$ (9), 205 (29), 193 (20), 189 (100), 161 (14), 131 (70), 130 (42), 115 (34), 91 (40), 57 (63), 41 (19). Anal. calcd. for $\mathrm{C}_{18} \mathrm{H}_{22} \mathrm{O}_{4}$ (302.4): C 71.50, H 7.33; Found: C 71.60, H $7.35 \%$.

Ethyl 5-(thiophen-2-yl)pent-2-enoate (4I). Yellow oil. ${ }^{1} \mathrm{H}$ NMR (300 $\left.\mathrm{MHz}, \mathrm{CDCl}_{3}\right)$ : $(E)$ isomer: $\delta 1.28\left(\mathrm{t},{ }^{3} J=7.2 \mathrm{~Hz}, 3 \mathrm{H}\right), 2.53-2.60(\mathrm{~m}, 2 \mathrm{H}), 2.97-3.02(2 \mathrm{H}), 4.18\left(\mathrm{q},{ }^{3} J=7.1 \mathrm{~Hz}, 2\right.$ H), $5.87\left(\mathrm{dt},{ }^{3} J=1.6,15.7 \mathrm{~Hz}, 1 \mathrm{H}\right), 6.80-6.82(\mathrm{~m}, 1 \mathrm{H}), 6.91-6.93(\mathrm{~m}, 1 \mathrm{H}), 7.00\left(\mathrm{dt},{ }^{3} J=6.8\right.$, $15.7 \mathrm{~Hz}, 1 \mathrm{H}), 7.12-7.14(\mathrm{~m}, 1 \mathrm{H})$; additional signals for the minor $(Z)$-isomer: $\delta 5.81\left(\mathrm{dt},{ }^{3} \mathrm{~J}=\right.$ $1.6,11.5 \mathrm{~Hz}, 1 \mathrm{H}), 6.24\left(\mathrm{dt},{ }^{3} \mathrm{~J}=7.2,11.5 \mathrm{~Hz}, 1 \mathrm{H}\right)$; additional signal for the minor $\alpha$-arylated isomer: $\delta 1.51\left(\mathrm{~d},{ }^{3} J=7.0 \mathrm{~Hz}, 1 \mathrm{H}\right) .{ }^{13} \mathrm{C} \mathrm{NMR}\left(75 \mathrm{MHz}, \mathrm{CDCl}_{3}\right): \delta 14.2\left(\mathrm{CH}_{3}\right), 28.4\left(\mathrm{CH}_{2}\right), 34.0$ $\left(\mathrm{CH}_{2}\right), 60.2\left(\mathrm{CH}_{2}\right), 122.3(\mathrm{CH}), 123.4(\mathrm{CH}), 124.5(\mathrm{CH}), 126.8(\mathrm{CH}), 143.4\left(\mathrm{C}_{\text {quat }}\right), 147.2(\mathrm{CH})$, $166.4\left(\mathrm{C}_{\text {quat }}\right)$. EI-MS (70 eV, m/z (\%)): $210\left(\mathrm{M}^{+}, 6\right), 165(8), 136(10), 97$ (100). Anal. calcd. for $\mathrm{C}_{11} \mathrm{H}_{14} \mathrm{O}_{2} \mathrm{~S}$ (210.3): C 62.83, H 6.71; Found: C 62.63, H 6.98\%.

Tert-butyl 2-(tert-butyl)-4-(5-ethoxy-5-oxopent-3-en-1-yl)-1H-pyrrole-1-carboxylate (4m). Yellow oil. ${ }^{1} \mathrm{H}$ NMR (300 MHz, $\left.\mathrm{CDCl}_{3}\right)$ : (E)-Isomer: $\delta 1.28\left(\mathrm{t},{ }^{3} J=7.2 \mathrm{~Hz}, 3 \mathrm{H}\right), 1.39(\mathrm{~s}, 9 \mathrm{H})$, 1.58 (s, $9 \mathrm{H}), 2.40-2.55(\mathrm{~m}, 4 \mathrm{H}), 4.19$ (q, $\left.{ }^{3} J=7.1,2 \mathrm{H}\right), 5.85\left(\mathrm{dt},{ }^{3} J=1.5,15.7 \mathrm{~Hz}, 1 \mathrm{H}\right), 5.91-$ $5.92(\mathrm{~m}, 1 \mathrm{H}), 6.96-7.05(\mathrm{~m}, 2 \mathrm{H})$; additional signals for the minor $(Z)$-Isomer: $\delta 1.28\left(\mathrm{t},{ }^{3} \mathrm{~J}=7.1\right.$ $\mathrm{Hz}, 3 \mathrm{H}), 2.47-2.52(\mathrm{~m}, 2 \mathrm{H}), 2.86-2.94(\mathrm{~m}, 2 \mathrm{H}), 4.15$ (q, $\left.{ }^{3} J=7.1 \mathrm{~Hz}, 2 \mathrm{H}\right), 5.77$ (dt, ${ }^{3} J=1.7$, $11.5 \mathrm{~Hz}, 1 \mathrm{H}), 6.24\left(\mathrm{dt},{ }^{3} \mathrm{~J}=7.3,11.5 \mathrm{~Hz}, 1 \mathrm{H}\right) .{ }^{13} \mathrm{C} \mathrm{NMR}\left(75 \mathrm{MHz}, \mathrm{CDCl}_{3}\right): \delta 14.3\left(\mathrm{CH}_{3}\right), 25.4$ $\left(\mathrm{CH}_{2}\right), 28.0\left(\mathrm{CH}_{3}\right), 29.9\left(\mathrm{CH}_{3}\right), 32.7\left(\mathrm{CH}_{2}\right), 33.2\left(\mathrm{C}_{\text {quat }}\right), 60.2\left(\mathrm{CH}_{2}\right), 82.8\left(\mathrm{C}_{\text {quat }}\right), 111.3(\mathrm{CH})$, $119.8(\mathrm{CH}), 121.6(\mathrm{CH}), 122.8\left(\mathrm{C}_{\text {quat }}\right), 145.2\left(\mathrm{C}_{\text {quat }}\right), 148.6(\mathrm{CH}), 149.2\left(\mathrm{C}_{\text {quat }}\right), 166.7\left(\mathrm{C}_{\text {quat }}\right)$. EIMS (70 eV, m/z(\%)): $349\left(\mathrm{M}^{+}, 6\right), 234$ (18), 136 (70), 57 (100), 43 (31). Anal. calcd. for $\mathrm{C}_{20} \mathrm{H}_{31} \mathrm{NO}_{4}$ (349.5): C 68.74, H 8.94, N 4.01; Found: C 68.69, H 9.21, N 3.82\%.

Tert-butyl 3-(5-ethoxy-5-oxopent-3-en-1-yl)-1H-indole-1-carboxylate (4n). Yellow oil. ${ }^{1} \mathrm{H}$ NMR (300 MHz, $\mathrm{CDCl}_{3}$ ): (E)-isomer: $\delta 1.29\left(\mathrm{t},{ }^{3} J=7.2 \mathrm{~Hz}, 3 \mathrm{H}\right), 1.67(\mathrm{~s}, 9 \mathrm{H}), 2.59-2.66(\mathrm{~m}, 2$ $\mathrm{H}), 2.83-2.88(\mathrm{~m}, 2 \mathrm{H}), 4.20\left(\mathrm{q},{ }^{3} J=7.1 \mathrm{~Hz}, 2 \mathrm{H}\right), 5.90\left(\mathrm{dt},{ }^{3} \mathrm{~J}=1.6,15.6 \mathrm{~Hz}, 1 \mathrm{H}\right), 7.05\left(\mathrm{dt},{ }^{3} \mathrm{~J}=\right.$ 6.8, 15.7 Hz, $1 \mathrm{H}), 7.21-7.40(\mathrm{~m}, 3 \mathrm{H}), 7.49-7.51(\mathrm{~m}, 1 \mathrm{H}), 8.11-8.14(\mathrm{~m}, 1 \mathrm{H})$; additional signal for the minor $(Z)$-isomer: $\delta 6.29\left(\mathrm{dt},{ }^{3} J=7.3,11.5,1 \mathrm{H}\right)$; additional signal for the minor $\alpha$-arylated isomer: $\delta 1.53\left(\mathrm{~d},{ }^{3} J=7.0 \mathrm{~Hz}, 1 \mathrm{H}\right) .{ }^{13} \mathrm{C} \mathrm{NMR}\left(75 \mathrm{MHz}, \mathrm{CDCl}_{3}\right): \delta 14.25\left(\mathrm{CH}_{3}\right)$, $23.58\left(\mathrm{CH}_{2}\right), 28.21\left(\mathrm{CH}_{3}\right), 31.72\left(\mathrm{CH}_{2}\right), 60.22\left(\mathrm{CH}_{2}\right), 83.45\left(\mathrm{C}_{\text {quat }}\right), 115.30(\mathrm{CH}), 118.76(\mathrm{CH})$, $119.62\left(\mathrm{C}_{\text {quat }}\right), 121.98(\mathrm{CH}), 122.37(\mathrm{CH}), 122.52(\mathrm{CH}), 124.38(\mathrm{CH}), 130.35\left(\mathrm{C}_{\text {quat }}\right), 147.96$ 
$(\mathrm{CH}), 149.75\left(\mathrm{C}_{\text {quat }}\right), 151.34\left(\mathrm{C}_{\text {quat }}\right), 166.54\left(\mathrm{C}_{\text {quat }}\right)$. EI-MS (70 eV, $\left.\mathrm{m} / \mathrm{z}(\%)\right): 343\left(\mathrm{M}^{+}, 6\right), 130$ (100), 57 (26). Anal. calcd. for $\mathrm{C}_{20} \mathrm{H}_{25} \mathrm{NO}_{4}$ (343.4): C 69.95, H 7.34, N 4.08; Found: C 69.88, H $7.44, \mathrm{~N} 4.02 \%$.

Tert-butyl 4-(5-ethoxy-5-oxopent-3-en-1-yl)-2-(thiophen-2-yl)-1 $H$-pyrrole-1-carboxylate (4o). Yellow oil. ${ }^{1} \mathrm{H}$ NMR (300 MHz, $\left.\mathrm{CDCl}_{3}\right)$ : (E)-isomer: $\delta 1.29\left(\mathrm{t},{ }^{3} \mathrm{~J}=7.1 \mathrm{~Hz}, 3 \mathrm{H}\right), 1.41(\mathrm{~s}, 9$ $\mathrm{H}), 2.45-2.52(\mathrm{~m}, 2 \mathrm{H}), 2.55-2.61(\mathrm{~m}, 2 \mathrm{H}), 4.19\left(\mathrm{q},{ }^{3} J=7.1 \mathrm{~Hz}, 2 \mathrm{H}\right), 5.87\left(\mathrm{dt},{ }^{3} J=1.5,15.7 \mathrm{~Hz}\right.$, $1 \mathrm{H})$, 6.18-6.20 (m, $1 \mathrm{H}), 6.96-7.07(\mathrm{~m}, 3 \mathrm{H}), 7.13-7.15(\mathrm{~m}, 1 \mathrm{H})$, 7.26-7.30 (m, $1 \mathrm{H})$; additional signals for the minor $(Z)$-isomer: $\delta 5.79\left(\mathrm{dt},{ }^{3} J=1.4,9.5 \mathrm{~Hz}, 1 \mathrm{H}\right), 6.26\left(\mathrm{dt},{ }^{3} J=4.2,11.5 \mathrm{~Hz}\right.$, $1 \mathrm{H}) .{ }^{13} \mathrm{C} \mathrm{NMR}\left(75 \mathrm{MHz}, \mathrm{CDCl}_{3}\right): \delta 14.24\left(\mathrm{CH}_{3}\right), 25.25\left(\mathrm{CH}_{2}\right), 27.65\left(\mathrm{CH}_{3}\right), 32.63\left(\mathrm{CH}_{2}\right), 60.18$ $\left(\mathrm{CH}_{2}\right), 83.54\left(\mathrm{C}_{\text {quat }}\right), 117.14(\mathrm{CH}), 119.90(\mathrm{CH}), 121.81(\mathrm{CH}), 124.54\left(\mathrm{C}_{\text {quat }}\right), 125.44(\mathrm{CH})$, $126.34(\mathrm{CH}), 127\left(\mathrm{C}_{\text {quat }}\right), 127.61(\mathrm{CH}), 134.85\left(\mathrm{C}_{\text {quat }}\right), 148.15(\mathrm{CH}), 148.90\left(\mathrm{C}_{\text {quat }}\right), 166.59$ (C $\left.\mathrm{C}_{\text {quat }}\right)$. EI-MS (70 eV, m/z (\%)): $375\left(\mathrm{M}^{+}, 10\right), 275$ (36), 202 (14), 162 (100), 57 (10). Anal. calcd. for $\mathrm{C}_{20} \mathrm{H}_{25} \mathrm{NO}_{4} \mathrm{~S}$ (375.5): C 63.97, H 6.71, N 3.73; Found: C 63.76, H 6.68, N 3.71\%.

Diethyl 5,5'-(10-hexyl-10H-phenothiazine-3,7-diyl)bis(pent-2-enoate) (4p). Yellow oil. ${ }^{1} \mathrm{H}$ NMR $\left(500 \mathrm{MHz}, \mathrm{CDCl}_{3}\right)$ : $(E, E)$-isomer: $\delta 0.87\left(\mathrm{t},{ }^{3} J=7.0 \mathrm{~Hz}, 3 \mathrm{H}\right), 1.24-1.33(\mathrm{~m}, 12 \mathrm{H}), 1.38$ 1.42 (m, $2 \mathrm{H}), 2.43-2.48$ (m, $4 \mathrm{H}), 2.64-2.67(\mathrm{~m}, 4 \mathrm{H}), 3.78\left(\mathrm{t},{ }^{3} J=7.1 \mathrm{~Hz}, 2 \mathrm{H}\right), 4.18$ (q, ${ }^{3} J=7.1$ $\mathrm{Hz}, 4 \mathrm{H}), 5.83\left(\mathrm{dt},{ }^{3} \mathrm{~J}=1.3,{ }^{3} \mathrm{~J}=15.6 \mathrm{~Hz}, 2 \mathrm{H}\right), 6.76-6.79(\mathrm{~m}, 2 \mathrm{H}), 6.92-7.00(\mathrm{~m}, 6 \mathrm{H})$; additional signal for the minor $(E, Z)$-isomer: $\delta 6.20\left(\mathrm{dt},{ }^{3} J=7.4,11.5 \mathrm{~Hz}, 1 \mathrm{H}\right)$; additional signal for the minor $\alpha$-arylated isomer: $\delta 1.36\left(\mathrm{~d},{ }^{3} J=7.0 \mathrm{~Hz}, 1 \mathrm{H}\right),{ }^{13} \mathrm{C} \mathrm{NMR}\left(126 \mathrm{MHz}, \mathrm{CDCl}_{3}\right): \delta 13.4$ $\left(\mathrm{CH}_{3}\right), 14.2\left(\mathrm{CH}_{3}\right), 22.6\left(\mathrm{CH}_{2}\right), 26.7\left(\mathrm{CH}_{2}\right), 26.8\left(\mathrm{CH}_{2}\right), 31.4\left(\mathrm{CH}_{2}\right) 33.3\left(\mathrm{CH}_{2}\right), 33.8\left(\mathrm{CH}_{2}\right), 60.2$ $\left(\mathrm{CH}_{2}\right), 115.2(\mathrm{CH}), 121.8(\mathrm{CH}), 127.1(2 \mathrm{CH}), 127.1\left(\mathrm{C}_{\text {quat }}\right), 134.8\left(\mathrm{C}_{\text {quat }}\right), 143.4\left(\mathrm{C}_{\text {quat }}\right), 147.9$ $(\mathrm{CH}), 166.5\left(\mathrm{C}_{\text {quat }}\right)$. EI-MS (70 eV, m/z (\%)): $535\left(\mathrm{M}^{+}, 38\right), 490$ (2), 450 (6), 422 (38), 339 (15), 325 (17), 278 (20), 277 (50), 254 (26), 224 (22), 94 (100). Anal. calcd. for $\mathrm{C}_{32} \mathrm{H}_{41} \mathrm{NO}_{4} \mathrm{~S}$ (535.7): C 71.74, H 7.71, N 2.61; Found: C 71.55, H 7.89, N 2.36\%.

\section{Acknowledgments}

The work was supported by the Fonds der Chemischen Industrie and the authors gratefully thank Merck Serono, Darmstadt, for the donation of chemicals.

\section{References}

1. For a monography, see Zhu, J.; Bienaymé, H., eds. Multicomponent Reactions. Wiley-VCH, Weinheim, 2005.

2. For reviews, see e.g. (a) Touré, B. B.; Hall, D. G. Chem. Rev. 2009, 109, 4439. (b) Sunderhaus, J. D.; Martin, S. F. Chem. Eur. J. 2009, 15, 1300. (c) Isambert, N.; Lavilla, R. Chem. Eur. J. 2008, 14, 8444. (d) Dömling, A. Chem. Rev. 2006, 106, 17. (e) Orru, R. V. A.; de Greef, M. Synthesis 2003, 1471. (f) Bienaymé, H.; Hulme, C.; Oddon, G.; Schmitt, P. 
Chem. Eur. J. 2000, 6, 3321. (g) Dömling, A.; Ugi, I. Angew. Chem. Int. Ed. Engl. 2000, 39, 3168. (h) Ugi, I.; Dömling, A.; Werner, B. J. Heterocycl. Chem. 2000, 37, 647. (i) Weber, L.; Illgen, K.; Almstetter, M. Synlett 1999, 366. (j) Armstrong, R. W.; Combs, A. P.; Tempest, P. A.; Brown, S. D.; Keating, T. A. Acc. Chem. Res. 1996, 29, 123. (k) Ugi, I.; Dömling, A.; Hörl, W. Endeavour 1994, 18, 115. (1) Posner, G. H. Chem. Rev. 1986, 86, 831.

3. For reviews, see e.g. (a) Tietze, L. F. Chem. Rev. 1996, 96, 115. (b) Tietze, L. F.; Beifuss, U. Angew. Chem. Int. Ed. Engl. 1993, 32, 131. (c) Tietze, L. F. J. Heterocycl. Chem. 1990, 27, 47.

4. Coquerel, Y.; Boddaert, T.; Presset, M.; Mailhol, D.; Rodriguez, J. In Ideas in Chemistry and Molecular Sciences: Advances in Synthetic Chemistry; Pignataro, B., Ed.; Wiley-VCH, Weinheim, 2010.

5. Vries, J. G. Can. J. Chem. 2001, 79, 1086.

6. Pommer, H. Angew. Chem. Int. Ed. Engl. 1977, 16, 423.

7. (a) Burke, M. D.; Schreiber, S. L. Angew. Chem. Int. Ed. 2004, 43, 46. (b) Burke, M. D.; Berger, E. M.; Schreiber, S. L. Science 2003, 302, 613. (c) Arya, P.; Chou, D. T. H.; Baek, M. G. Angew. Chem. Int. Ed. 2001, 40, 339. (d) Cox, B.; Denyer, J. C.; Binnie, A.; Donnelly, M. C.; Evans, B.; Green, D. V. S.; Lewis, J. A.; Mander, T. H.; Merritt, A. T.; Valler, M. J.; Watson, S. P. Progr. Med. Chem. 2000, 37, 83. (e) Schreiber, S. L. Science 2000, 287, 1964.

8. For a monography, see e.g. Oestreich, M., Ed. Mizoroki-Heck Reaction. Wiley-VCH, Weinheim, 2009.

9. For representative reviews, see e.g. (a) Coeffard, V.; Guiry, P. J. Curr. Org. Chem. 2010, 14, 212. (b) Rossi, R.; Bellina, F.; Lessi, M. Synthesis 2010, 4131. (c) Alonso, F.; Beletskaya, I. P.; Yus, M. Tetrahedron 2005, 61, 11771. (d) Bräse, S.; de Meijere, A. In Metal-Catalyzed Cross-Coupling Reactions. De Meijere, A.; Diederich, F., Eds.; WileyVCH, Weinheim, 2004, Vol. 1, p 217. (e) Beletskaya, I. P.; Cheprakov, A. V. Chem. Rev. 2000, 100, 3009. (f) de Meijere, A.; Meyer, F. E. Angew. Chem. Int. Ed. Engl. 1994, 33, 2379.

10. (a) Maryanoff, B. E.; Reitz, A. B. Chem. Rev. 1989, 89, 863. (b) Nicolaou, K. C.; Harter, M. W.; Gunzner, J. L.; Nadin, A. Liebigs Ann. Recueil 1997, 1283.

11. Burmester, C.; Mataka, S.; Thiemann, T. Synth. Commun. 2010, 40, 3196. (b) Saiyed, A. S.; Bedekar, A. V. Tetrahedron Lett. 2010, 51, 6227.

12. For a review on Pd-catalyzed reactions with unsaturated alcohols, see e.g. Muzart, J. Tetrahedron 2005, 61, 4179. (b) Melpolder, J. B.; Heck, R. F. J. Org. Chem. 1976, 41, 265.

13. Kressierer, C. J.; Müller, T. J. J. Angew. Chem. Int. Ed. 2004, 43, 5997.

14. Körber, N.; Rominger, F.; Müller, T. J. J. Synlett 2010, 782.

15. Shen, Y.; Xin, Y.; Zhao, J. Tetrahedron Lett. 1988, 29, 6119.

16. Huo, H.; He, X.; Chan, T. H. J. Org. Chem. 2008, 73, 8583. 
17. (a) Jeffery, T. Tetrahedron 1996, 52, 10113. (b) Jeffery, T. J. Chem. Soc., Chem. Commun. $1984,19,1287$.

18. The ethyl 5-(hetero)arylpent-2-enoates 4 were isolated as a mixture of regio- and stereoisomers (as indicated by the appearance of signal sets of diagnostic proton and carbon resonances), however, the depicted structures are the major $\beta$-regioisomers with predominant $E$-configuration of the double bond.

19. Kamal, A.; Krishnaji, T.; Reddy, P. V. Tetrahedron Asymm. 2007, 18, 1775.

20. Chu, L.; Hutchins J. E.; Weber A. E.; Jo, J. L.; Yang, Y. T.; Cheng, K.; Smith, R. G.; Fisher, M. H.;Wyvratt, M. J.; Goulet, M.T. Bioorg. Med. Chem. Lett. 2001, 11, 509.

21. Merschaert, A.; Delbeke, P.; Daloze, D.; Dive, G. Tetrahedron Lett. 2004, 45, 4697.

22. Bunce, R. A.; Lee, E. J. J. Heterocyclic Chem. 2010, 47, 1176.

23. Barrow, R. A.; Hemscheidt,T.; Liang, J.; Paik, S.; Moore, R. E.; Tius, M. A. J. Am. Chem. Soc. 1995, 117, 2479.

24. Levin, J. I.; Weinreb, S. M. J. Am. Chem. Soc. 1983, 105, 1397.

25. Abell, A. D.; Hoult, D. A.; Bergman, D. A.; Fairlie, D. P. Bioorg. Med. Chem. Lett. 1997, 7, 2853.

26. Azizi, N.; Saidi, M. R. Tetrahedron 2004, 60, 383.

27. Jonsson, S. Y.; Adolfsson, H.; Bäckvall, J. E. Chem. Eur. J. 2003, 9, 2783.

28. Shinohara, I.; Okue, M.; Yamada, Y.; Nagaoka, H. Tetrahedron Lett. 2003, 44, 4649.

29. Zhu, H.-J.; Pittman, C. U. Synth. Commun. 2003, 33, 1733.

30. Merkul, E.; Boersch, C.; Frank, W.; Müller, T. J. J. Org. Lett. 2009, 11, 2269. 